Ergod. Th. \& Dynam. Sys. (1988), 8, 437-457

Printed in Great Britain

\title{
On ergodic foliations
}

\author{
KYEWON PARK \\ Department of Mathematics, Bryn Mawr College, Bryn Mawr, PA 19010, USA
}

(Received 2 March 1986 and revised 29 December 1986)

\begin{abstract}
We define an ergodic $\mathbb{Z}$-foliation and show that it can be realized as a quotient space of the 'covering space'. The covering space has two actions, $T$ and $S$, where $T$ is a $\mathbb{Z}$-action, $S$ is a map of order two, and $S$ and $T$ skew-commute; that is, $S T S=T^{-1}$. We study the isometry between two foliations via the isomorphism between two bigger group actions in the covering spaces. Properties of an ergodic foliation are studied in a way similar to the study of an ergodic action. We construct a counterexample of a $K$-automorphism to show that, unlike Bernoulli automorphisms, $\mathbb{Z}$-actions do not completely determine $\mathbb{Z}$-foliations.
\end{abstract}

\section{Introduction}

It is well known that the horocycles on the Poincaré disk have 'exotic' properties such as minimal self-joinings and being isomorphic to any rescaling of itself [7]. However, when we consider the Poincaré sphere, the horospherical foliation defines an equivalence relation in the sphere such that all equivalence classes are isometric. The difference between the disk and the sphere is that in the disk there is an ergodic action, denoted by $T_{t}$, such that the horocycles become orbits of the action, but in the case of the sphere the equivalence relation does not admit a measurable action such that equivalence classes become orbits of the action.

Before we define a measurable foliation rigorously, we want to discuss a concrete example of a measurable $\mathbb{Z}$-foliation constructed by D. Rudolph. A sketch of the construction is as follows:

Let $X$ be a set $[0,1) \times\{0,1\}$ where $[0,1)$ is an interval with Lebesgue measure. We denote the space with the obvious measure by $(X, \mathscr{F}, \mu)$. We say two points in $X, x=(\alpha, \omega)$ and $x^{\prime}=\left(\alpha^{\prime}, \omega^{\prime}\right)$, are equivalent if their first coordinates are the same. Divide this set into two subsets $B_{0,1}=\left[0, \frac{1}{2}\right) \times\{0,1\}$ and $B_{0,2}=\left[\frac{1}{2}, 1\right) \times\{0,1\}$. That is, $B_{0,1}$ is the left half and $B_{0,2}$ is the right half. We place $B_{0,2}$ upside down above $B_{0,1}$. (See figure 1.) We call this block $B_{1}$. This block can be regarded as $\left[0, \frac{1}{2}\right) \times\{0,1,2,3\}$. We say that two points in $B_{1}$ are equivalent if their first coordinates are the same. We note that the equivalence in $B_{2}$ respects that in $B_{1}$. If $x$ and $x^{\prime}$ are equivalent, we define the distance $d\left(x, x^{\prime}\right)=\left|\omega-\omega^{\prime}\right|$ where $\omega$ and $\omega^{\prime}$ denote the second coordinates of $x$ and $x^{\prime}$, respectively, in $B_{1}=\left[0, \frac{1}{2}\right) \times\{0,1,2,3\}$. To construct $B_{n+1}$ block from $B_{n}$, divide $B_{n}$ into two subsets, $B_{n, 1}$ and $B_{n, 2}$ where $B_{n, 1}$ is the left half of $B_{n}$ and $B_{n, 2}$ is the right half of $B_{n}$. Place the right half $B_{n, 2}$ upside down above the left 

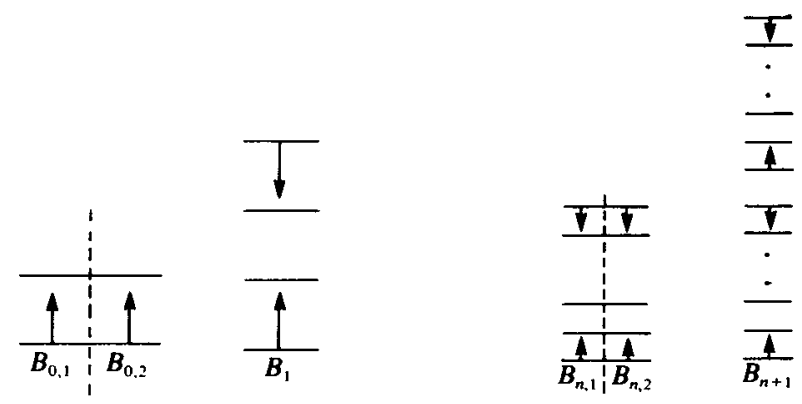

FIGURE 1

half $B_{n, 1}$. This $B_{n+1}$ block can be considered as $\left[0,\left(\frac{1}{2}\right)^{n+1}\right) \times\left\{0,1,2, \ldots, 2^{n+2}-1\right\}$. We define two points in $B_{n+1}$ to be equivalent if their first coordinates are the same. If $x$ and $x^{\prime}$ are equivalent in $B_{n+1}$, then we define the distance between these two points to be $\left|\omega-\omega^{\prime}\right|$. It is clear that the equivalence relation and the metric in an equivalence class in $B_{n+1}$ is consistent with those in $B_{n}$, respectively. Let $(X, \mathscr{E}, \mathscr{F}, \mu)$ be the limit space of this construction. There is an obvious equivalence relation, denoted by $\mathscr{E}$, on $X$ such that each equivalence class with the metric measuring the gaps between any two points in the class is isometric to $\mathbb{Z}$. Hence we call this a $\mathbb{Z}$-foliation on $X$.

What we will see is that this $\mathbb{Z}$-foliation can not be 'realized' by a measurable action. That is, there is no measurable action $T$ in $(X, \mathscr{E}, \mathscr{F}, \mu)$ so that each equivalence class is an orbit under the action. We note that a $\mathbb{Z}$-action on a measure space defines an equivalence relation and each equivalence class (called an orbit) has not only a metric but a natural direction (an order). Suppose we have a measurable action $T$ on $(X, \mathscr{E}, \mathscr{F}, \mu)$ such that $T$ preserves the metric within an equivalence class. Hence every point $(t, i)$ in a level set of $B_{n}$ is mapped by $T$ to $(t, i+1)$ or $(t, i-1)$. Since the level sets of the $B_{n}$ 's generate the $\sigma$-algebra, there exists a level set $B_{k}^{i}=\left[0,\left(\frac{1}{2}\right)^{k+1}\right) \times\{i\}$ for some $k$ and for some $i \in\left\{0,1,2, \ldots, 2^{k+2}-1\right\}$ such that $\mu\{(t, i) ; T(t, i)=(t, i+1)\} \geq 0.99 \mu B_{k}^{i}$.

Hence we can say that at least $98 \%$ of each of the right and the left halves of $B_{k}^{i}$ are mapped by $T$ to the points just above themselves. Since the right half of $B_{k}^{i}$ is placed upside down above the left half at the next step, we have

$$
T\left(T^{2^{k+1}-2 i-1}(t, i)\right)=T\left(t, 2^{k-1}-i-1\right)=\left(t, 2^{k+2}-i\right)
$$

for the $98 \%$ of $(t, i)$ 's in the left half of $B_{k}^{i}$. Since $T^{2^{k+1}-2 i-1}(t, i)$ is the right half of $B_{k}^{i}$, we have

$$
T\left(T^{2^{k+1}-2 i-1}(t, i)\right)=\left(t, 2^{k+1}-2 i-2\right)
$$

for the $98 \%$ of $(t, i)$ 's in the left half of $B_{k}^{i}$. Certainly (1) and (2) lead to a contradiction.

It is clear from our argument that if we start to place the right half of $B_{n}$ above the left half (not upside down), then there exists a $\mathbb{Z}$-action $T$ (an adding machine) such that each equivalence class becomes an orbit of $T$. The example of an ergodic foliation in the Poincare sphere which is mentioned at the beginning of our introduction has been further studied by L. Flaminio.[2] 
We will divide the paper into two parts. In part I, we study an ergodic foliation; in part II, we construct an example of a $K$-automorphism which shows that, unlike a Bernoulli automorphism, a $\mathbb{Z}$-action does not completely determine a foliation.

I would like to thank Professor D. Ornstein and D. Rudolph for their helpful discussions.

This research was supported in part by a Bryn Mawr Faculty Research Grant and NSF Grant DMS-8604202.

\section{Part I. An ergodic foliation.}

1. Definitions and Preliminaries.

Let $(X, \mathscr{F}, \mu)$ be a Lebesgue space. An ergodic foliation can be regarded as a generalized ergodic action in the sense that we require an action to be defined on a proper subset (that is, locally) instead of on the whole set $X$ to itself. An ergodic action in $(X, F, \mu)$ obviously gives rise to an ergodic foliation.

Let $E$ be a subset of $X$. We define a measure $\nu$ on the product $\sigma$-algebra of $E \times\{-1,0,1\}$ so that $\nu(F \times\{l\})=\mu F$ for all measurable subset $F \subset E$ and $l=-1$, 0 or 1 .

Definition 1. We define a local action $T$, sometimes denoted by $T_{E}$, on a subset $E \subset X$ to be a one-to-one, bimeasurable and measure perserving map from $E \times$ $\{-1,0,1\}$ to $X$, satisfying $T(E \times\{0\})=E$.

We denote $T(x, l)$ by $T^{\prime}(x)$. Since $T_{E}$ is measure preserving, $\left\{T^{-1} E, E, T E\right\}$ are pairwise disjoint and we call this a local chart. We also call the $\operatorname{set}\left\{T^{-1} x, x, T x\right\}$ a leaf of $x \in E$ generated by $T_{E}$.

We may remark that we used the term 'local' in two seemingly different meanings. The first is in the sense that $T$ is defined on a proper subset $E$ of $X$ and the second is in the sense that it is defined on $\{-1,0,1\}$, a proper subset of $\mathbb{Z}$.

Definition 2. We say that a Lebesgue space $(X, \mathscr{F}, \mu)$ has a foliation $(X, \mathscr{E}, \mathscr{F}, \mu)$ if there exists a countable collection of subsets $\left\{E_{i}\right\}$ such that

(i) $\bigcup E_{i}=X$

(ii) There exists a local action $T_{E_{i}}$ for each $E_{i}$. (We will write $T_{i}(x)$ for $T_{E_{i}}(x)$ for convenience of notation.)

(iii) If $x \in E_{i} \cap E_{j}$, then two leaves of $x$ generated by $T_{i}$ and $T_{j}$ are the same.

If $x \in E_{i} \cap T_{j}^{l}\left(E_{j}\right)$ for some $E_{j}$ and some $l=-1$ or 1 , then there exists some $l^{\prime}=-1$ or 1 such that $T_{j}^{l}\left(T_{i}^{l^{\prime}}(x)\right)=x$.

We denote by $U_{i}$ the local chart generated by $E_{i}$. We remark here that this definition of an ergodic foliation is similar to the one given by $\mathbf{R}$. Zimmer. [11]

Definition 3. We say $x$ is equivalent to $y(x \sim y)$ in $(X, \mathscr{E}, \mathscr{F}, \mu)$ if and only if there exists a finite subset $\left\{x=x_{0}, x_{1}, x_{2}, \ldots, x_{n-1}, x_{n}=y\right\}$ such that

$$
x_{1}=T_{i_{0}}^{l}\left(x_{0}\right), \quad x_{2}=T_{i_{1}}^{l_{1}}\left(x_{1}\right), \ldots, y=x_{n}=T_{i_{n-1}}^{l_{n-1}}\left(x_{n-1}\right) \quad \text { where } l_{i}=1 \text { or }-1
$$

It is clear that this is an equivalence relation in $(X, \mathscr{F}, \mu)$. We call the set $L(x)=$ $\{y \mid y \sim x\}$ the leaf of $x$. 
Definition 4. A foliation $(X, \mathscr{E}, \mathscr{F}, \mu)$ is called an ergodic $\mathbb{Z}$-foliation if and only if $\bigcup_{x \in A} L(x)=X$ for every subset $A$ of positive measure.

Clearly an ergodic $\mathbb{Z}$-action $(X, T, \mathscr{F}, \mu)$ gives rise to an ergodic foliation. We define a metric, $d$, in each leaf of an ergodic $\mathbb{Z}$-foliation such that if $x$ and $y$ are as in the definition 3 , then $d(x, y)=n$. This metric measures how many points there are between $x$ and $y$. We say a foliation is aperiodic if $\mu\{x ; \# L(x)<\infty\}=0$. Clearly an ergodic foliation is aperiodic unless $X$ differs from a finite set by a set of measure 0 .

Definition 5. Two ergodic foliations, $\left(X_{1}, \mathscr{E}_{1}, \mathscr{F}_{1}, \mu_{1}\right)$ and $\left(X_{2}, \mathscr{C}_{2}, \mathscr{F}_{2}, \mu_{2}\right)$, are leaf equivalent if and only if there exists a measure preserving and invertible map $\varphi$ from $\left(X_{1}, \mathscr{E}_{1}, \mathscr{F}_{1}, \mu_{1}\right)$ to $\left(X_{2}, \mathscr{C}_{2}, \mathscr{F}_{2}, \mu_{2}\right)$ such that $\varphi$ preserves the leaves.

Definition 6. Two ergodic foliations are isometric if and only if there exists an invertible and measure preserving map $\varphi$ from $\left(X_{1}, \mathscr{C}_{1}, \mathscr{F}_{1}, \mu_{1}\right)$ to $\left(X_{2}, \mathscr{C}_{2}, \mathscr{F}_{2}, \mu_{2}\right)$ such that $\varphi$ preserves the leaves and the metric within leaves, that is $d(x, y)=$ $d(\varphi(x), \varphi(y))$.

We may note that when two foliations arise from two ergodic transformations, say $T$ and $\tilde{T}$, it is clear that leaf-equivalence is orbit equivalence and the isometry between two spaces is an isomorphism between $T$ and $\tilde{T}$ or $T$ and $\tilde{T}^{-1}$.

Now that we have an isometry between foliations, we can also define Kakutani equivalence between two foliations. Let $A$ be a subset of an ergodic foliation $(X, \mathscr{E}, \mathscr{F}, \mu)$. There is a natural induced equivalence relation in $A$ together with the obvious induced metric on each equivalence class of $A$. We say two foliations are Kakutani equivalent if and only if there exist subsets $A_{1} \subset X_{1}$ and $A_{2} \subset X_{2}$ such that $A_{1}$ and $A_{2}$ with the induced equivalence relations and metrics respectively are isometric to each other.

We remark that all these definitions can be extended analogously to an $\mathbb{R}^{\prime}$-foliation and other bigger group-foliations.

\section{Representation of an ergodic $\mathbb{Z}$-foliation}

In this section, we will study the relation between an ergodic foliation and an ergodic action.

Lemma 1. Let $(X, \mathscr{E}, \mathscr{F}, \mu)$ be an ergodic $\mathbb{Z}$-foliation. There exists a subset $B$ and an integer valued measurable function $f(x)$ on $B$ such that $(X, \mathscr{E}, \mathscr{F}, \mu)$ is represented as $a$ skyscraper with $a$ base $B$ and $a$ height function $f(x)$.

Proof. We take an arbitrary subset $A$ of positive measure. Since $\cup E_{i}=X$, there exists $E_{i}$ containing $x \in A$ for every $x$. We start with $E_{1} \in\left\{E_{i}\right\}$. Let $F=A \cap E_{1}$. If $F=\varnothing$, then we consider $F=A \cap E_{2}$. We assume that $F \neq \varnothing$. Let $x$ be a point in $F$. If $T_{1}(x) \in A$, we do nothing. If $T_{1}(x) \notin A$, then place $T_{1}(x)$ just above $x$. If $T_{1}^{-1}(x) \in A$, then we do nothing. If $T_{1}^{-1}(x) \notin A$, then place $T_{1}^{-1}(x)$ just below $x$. After we do this for every $x \in F$, we will denote the resulting set $A_{1,1}=A \cup T_{1}\left(A \cap E_{1}\right) \cup T_{1}^{-1}\left(A \cap E_{1}\right)$, which is clearly measurable. We denote each level of $A_{1,1}$ by $A_{1,1}^{0}=A, A_{1,1}^{1}=$ $T_{1}\left(A \cap E_{1}\right)-A$ and $A_{1,1}^{-1}=T_{1}^{-1}\left(A \cap E_{1}\right)-A$. Next we consider $A_{1,1} \cap E_{2}$. If this is a 
null set, then we go to $A_{1,1} \cap E_{3}$. Otherwise for each $x \in A_{1,1} \cap E_{2}$, we repeat the previous steps with a little more care. That is, for $l=1$ or -1 ,

(i) If $T_{2}^{l}(x) \in A_{1,1}$, then do nothing;

(ii) if $T_{2}^{l}(x) \notin A_{1,1}$, then we consider two cases. In the case when $T_{2}^{-l}(x)$ is either above or below $x$, we place $T_{2}^{l}(x)$ above or below $x$ opposite from $T_{2}^{-l}(x)$. Otherwise we place $T_{2}^{l}(x)$ either above or below $x$ corresponding to $l=1$ or -1 respectively. We let $A_{1,2}=A_{1,1} \cup T_{2}\left(A_{1,1} \cap E_{2}\right) \cup T_{2}^{-1}\left(A_{1,1} \cap E_{2}\right)$. We denote each level set of $A_{1,2}$ as $A_{1,2}^{2}, A_{1,2}^{1}, A_{1,2}^{0}, A_{1,2}^{-1}$ and $A_{1,2}^{-2}$, respectively. We note that $A_{1,1}^{1} \subset A_{1,2}^{1}, A_{1,1}^{0}=A_{1,2}^{0}$ and $A_{1,1}^{-1} \subset A_{1,2}^{-1}$. We consider $A_{1,2} \cap E_{1}$ and repeat the above three steps (i), (ii) and (iii) for each $x \in A_{1,2} \cap E_{1}$. We call the resulting set

$$
A_{2,1}=A_{1,2} \cup T_{1}\left(A_{1,2} \cap E_{1}\right) \cup T_{1}^{-1}\left(A_{1,2} \cap E_{1}\right) \text {. }
$$

We repeat the above three steps for each point in $A_{2,1} \cap E_{2}$ and let

$$
A_{2,2}=A_{2,1} \cup T_{2}\left(A_{2,1} \cap E_{2}\right) \cup T_{2}^{-1}\left(A_{2,1} \cap E_{2}\right) \text {. }
$$

We define $A_{i, j}(i=1,2, \ldots, j=1,2, \ldots, i+1)$ inductively as

$$
\begin{aligned}
& A_{i, 1}=A_{i-1, i} \cup T_{1}\left(A_{i-1, i} \cap E_{1}\right) \cup T_{1}^{-1}\left(A_{i-1, i} \cap E_{1}\right) \\
& A_{i, j}=A_{i, j-1} \cup T_{j}\left(A_{i, j-1} \cap E_{j}\right) \cup T_{j}^{-1}\left(A_{i, j-1} \cap E_{j}\right) \quad j=2, \ldots, i+1
\end{aligned}
$$

We let $F_{i}=\bigcup_{j=1}^{i+1} A_{i, j}$. It is clear that each of the $F_{i}$ 's is a two sided tower built over $A$ and they are increasing sequence of towers.

We claim that $\bigcup_{i} F_{i}=X$. Let $y$ be any point in $X$. Since the foliation is ergodic, there exists a point, say $x$, in $A$ such that $x \sim y$. Let $\left\{x=x_{0}, x_{1}, \ldots, x_{n}=y\right\}$ be a set of all points between $x$ and $y$. For each $x_{i}$, let $E_{l_{i}}$ be the first $E_{j}$ that contains $x_{i}$ $(i=0,1, \ldots, n)$. From our construction, if $x_{0}$ is in $E_{1}$, then $x_{1}$ is in $A_{1,1}$ and if $x_{0}$ is in $E_{b_{0}}\left(l_{0} \neq 1\right)$, then $x_{1}$ is in $A_{l_{0}-1, l_{0}}$ at the latest. If $l_{i}<l_{i-1}$, then $x_{i+1}$ is in $A_{l_{i-1}, l_{i}} \cdot$ If $l_{i}>l_{i-1}$, then $x_{i+1}$ is in $A_{l_{i}-1, l_{i}}$. Therefore $\left\{x_{0}, x_{1}, \ldots, x_{n}=y\right\}$ are contained in $\bigcup_{i=1}^{\infty} \bigcup_{j=1}^{i+1} A_{i, j}$. In particular, $y \in \bigcup_{i=1}^{\infty} \bigcup_{j=1}^{i+1} A_{i, j}$.

We let $A^{k}=\lim _{i \rightarrow \infty} A_{i, 1}^{k}$ for $k=0, \pm 1, \pm 2, \ldots$ That is, $A^{k}$ denotes the $k$ th level set in $\bigcup_{i=1}^{\infty} \bigcup_{j=1}^{i+1} A_{i, j}$. We let $B=\left\{x \in X \mid\right.$ if $x \in A^{k}$, then there is no point $y \in A^{k-1}$ such that $y \sim x$ for all $k\}$. [See figure 2.] Clearly, $\mu B=\mu A$. We let $B_{k}=$ $\left\{x \in B \mid x \in A^{k}, k=0,-1,-2, \ldots\right\}$. We note that $B=\bigcup B_{k}$. Let $B_{k}^{l}=\left\{y \mid y \in A^{k+1}\right.$ and $y \sim x$ for some $\left.x \in B_{k}\right\}$ for $l=0,1,2, \ldots$ and $k=0,-1,-2, \ldots$ Let $B^{l}=\bigcup_{k=0}^{-\infty} B_{k}^{l}$. Clearly $\bigcup_{l=0}^{\infty} B^{\prime}=X=\bigcup_{i=1}^{\infty} \bigcup_{j=1}^{i+1} A_{i, j}$ and $\left\{B^{l}\right\}$ is the desired skyscraper built over $B$. This completes the proof of the Lemma.

We call this $B$ the base and $B^{l}$ the $l$ th level set above $B$. We mention that each level set is clearly measurable and the function $f(x)$ could be unbounded. The difference between this skyscraper of a foliation and that of an ergodic action is that the points which do not have points directly above them are not necessarily mapped by the local action to the points on the bottom level of the tower. Some of the points could be mapped to the points which do not have points directly above them.

The following lemma is the Rokhlin Lemma for an ergodic foliation.

Lemma 2. Given $N$ and $\varepsilon>0$, there exists a Rokhlin tower built over a set, $B$, such that each point $x$ in $B$ has the constant height $N$ and $\mu\left(\bigcup_{i=0}^{N-1} B^{i}\right)>1-\varepsilon$ where $B^{i}$ is the ith level set above $B$ for $i=0,1,2, \ldots, N-1$. 


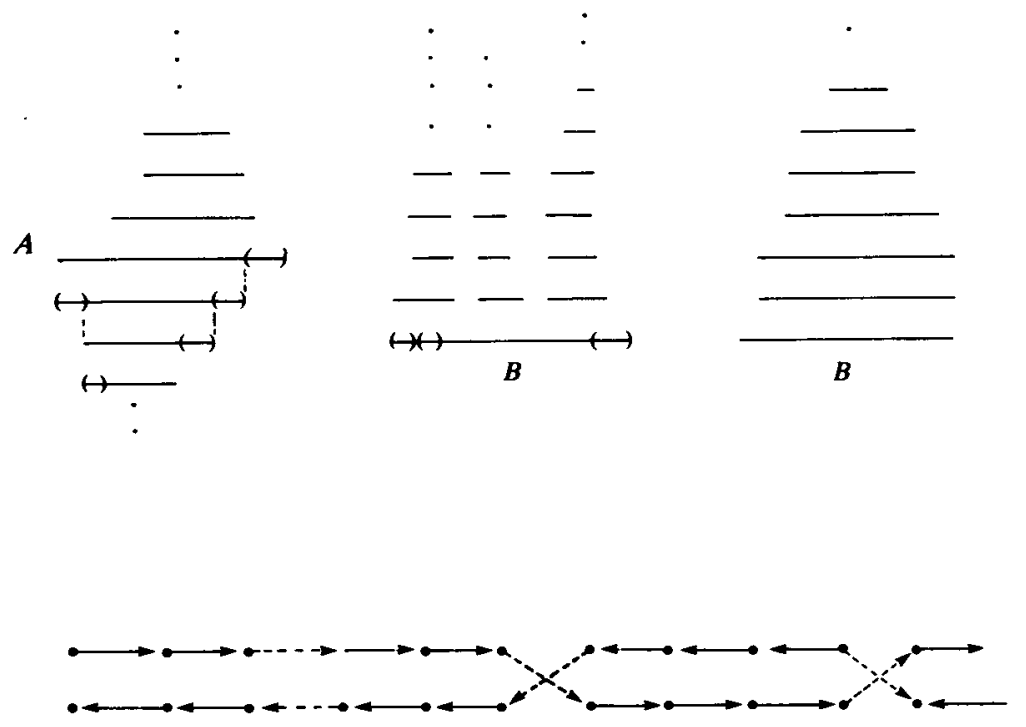

FIGURE 2

Proof. Choose $\delta$ such that $\delta \cdot N<\varepsilon / 2$. Consider the tower built over $E$ whose measure is less than $\delta$ (We can do this by taking the set $A$ in Lemma 1 to have a measure smaller than $\delta$ ). Let $E^{i}$ denote the $i$ th level set above $E$. Since $\mu\left(\bigcup_{i=0}^{\infty} E^{i}\right)=$ 1 , there exists $t$ so that $\mu\left(\bigcup_{i=0}^{t N-1} E^{i}\right)>1-\varepsilon / 2$. Let $D^{0}=\{x \in E$ : there exists a point $y \in E^{i}$ such that $d(x, y)=i$ for each $\left.i=0,1, \ldots, N-1\right\}$. Likewise, we define $D^{k}=$ $\left\{x \in E^{N \cdot k}\right.$ : there exists a point $y \in E^{N \cdot k+i}$ such that $d(x, y)=i$ for each $i=0$, $1, \ldots, N-1\}$. We let $B=\bigcup_{k=0}^{t-1} D^{k}$. Now we have

$$
\begin{aligned}
\mu\left(\bigcup_{i=0}^{N-1} B^{i}\right) & =\sum_{k=0}^{i-1} N \cdot \mu\left(D^{k}\right) \\
& \geq 1-\varepsilon / 2-\sum_{k=0}^{t-1} N \cdot \mu\left(E^{N \cdot k}-D^{k}\right) \\
& =1-\varepsilon / 2-N \cdot \mu\left(\bigcup_{k=0}^{i-1}\left(E^{N \cdot k}-D^{k}\right)\right) \geq 1-\varepsilon / 2-N \cdot \mu(B) \\
& \geq 1-\varepsilon .
\end{aligned}
$$

We remark that analogous definitions and lemmas hold true for an ergodic $\mathbb{R}^{1}$ foliation. Lemma 1 for an ergodic $\mathbb{R}^{1}$-foliation corresponds to Ambrose's Representation Theorem for an ergodic flow. [1]

Using Lemma 1 above, we will construct a covering space of a foliation.

THEOREM 1. If $(X, \mathscr{E}, \mu, \mathscr{F})$ is an ergodic $\mathbb{Z}$-foliation, then there exists a measure space $(\hat{X}, \hat{\mu}, \hat{\mathscr{F}})$ with a $\mathbb{Z}$-action $T$ and an order two map $S$ such that $S T S=T^{-1}$.

Proof. We assume that the foliation $(X, \mathscr{E}, \mu, \mathscr{F})$ is represented as a skyscraper over a base $B$. To make our definition of $T$ clear, we may assume that $\mu\left(B^{1}\right)=\mu(B)$, that is, every point in $B$ has a point directly above it. We attach an arrow pointing 
upward to every point of the skyscraper except those which do not have points directly above. Let $\left(X_{1}, \mathscr{C}_{1}, \mu_{1}, \mathscr{F}_{1}\right)$ be a copy of the skyscraper and $\left(X_{2}, \mathscr{C}_{2}, \mu_{2}, \mathscr{F}_{2}\right)$ be another copy of the skyscraper with arrows pointing downwards at every point except those on $B$. Let $\hat{X}=X_{1} \cup X_{2}$ with the obvious $\sigma$-algebra and the measure. Given $x \in X$, we let $x_{1} \in X$ and $x_{2} \in X_{2}$ be two corresponding points in $\hat{X}$. We define $T$ on $\hat{X}$ as follows (See figure 2). First we define $T$ on $X_{1}$. If there is an arrow (a direction) coming out from $x_{1}$, then we define $T\left(x_{1}\right)=x_{1}^{\prime}$ where $x_{1}^{\prime}$ is the point whose distance from $x_{1}$ is 1 in the given direction. If $x_{1}$ is a point on the skyscraper which does not have a point above it (there is no arrow coming out from $x_{1}$ ), then we will consider two cases separately. Since $T$ is well defined on every point of $B$, we note that $T^{-1}\left(x_{1}\right)$ is well defined and $d\left(x_{1}, T^{-1}\left(x_{1}\right)\right)=1$. We denote by $x_{1}$ the other point whose distance from $x_{1}$ is 1 .

Case 1. If there is the arrow coming out from $x_{1}^{\prime}$, then we define $T\left(x_{1}\right)=x_{1}^{\prime}$.

Case 2. If there is no arrow coming out from $x_{1}^{\prime}$, then we define $T\left(x_{1}\right)=x_{2}^{\prime}$ where $x_{2}^{\prime}$ in $X_{2}$ is the point corresponding to $x_{1}^{\prime}$ in $X_{1}$.

We note that there are no three consecutive points without arrows between any two points with arrows. We define $T$ on $X_{2}$ analogously. If $x_{2}$ is a point from which an arrow (a direction) is coming out, then we define $T\left(x_{2}\right)=x_{2}^{\prime}$ where $x_{2}^{\prime}$ is the point whose distance from $x_{1}$ is 1 in the given direction. If $x_{2}$ is a point from which no arrow is coming out (that is, $x_{2}$ is on the base of the tower of $\left.X_{2}\right), T^{-1}\left(x_{2}\right)$ is well defined and $d\left(x_{2}, T^{-1}\left(x_{2}\right)\right)=1$. We consider two cases as before. We denote by $x_{2}^{\prime}$ the other point whose distance from $x_{2}$ is 1 .

Case 1. If there is an arrow coming out from $x_{2}^{\prime}$, then we define $T\left(x_{2}\right)=x_{2}^{\prime}$.

Case 2. If there is no arrow coming out from $x_{2}^{\prime}$, then we define $T\left(x_{2}\right)=x_{1}^{\prime}$ where $x_{1}^{\prime}$ in $X_{1}$ is the corresponding point to $x_{2}^{\prime}$ in $X_{2}$.

It is not hard to see that $T$ is a measurable $\mathbb{Z}$-action. If we define $S\left(x_{1}\right)=x_{2}$ and $S\left(x_{2}\right)=x_{1}$, then it is clear that $S$ satisfies

(i) $S^{2}=1$

(ii) $S T S=T^{-1}$ ( $S$ skew commutes with $T$; $T$ and $T^{-1}$ are isomorphic by an order two map.)

THEOREM 2. Let $(\hat{X},\{T, S\}, \hat{\mu}, \hat{\mathscr{F}})$ be a space where $T$ is an ergodic $\mathbb{Z}$-action and $S$ is a map of order two which skew-commutes with $T$. The quotient space by identifying $x$ and $S(x)$ gives rise to a foliation $(X, \mathscr{E}, \mu, \mathscr{F})$ with the quotient $\sigma$-algebra.

Proof. Since this is a straight measure theory, we will skip the details. Let $\mathcal{M}$ be a collection of measurable subsets $\left\{\hat{E}_{\alpha}\right\}$ where each $\hat{E}_{\alpha}$ satisfies that $\left\{T \hat{E}_{\alpha}, \hat{E}_{\alpha}, T^{-1} \hat{E}_{\alpha}, S T \hat{E}_{\alpha}, S \hat{E}_{\alpha}, S T^{-1} \hat{E}_{\alpha}\right\}$ are pairwise disjoint. It is not hard to show that for every subset $A$ of positive measure, there exists a subset $A_{0}(\subset A)$ in $\mathcal{M}$. Hence $M$ is non-empty. We claim that there exists a countable subcollection $\left\{\hat{E}_{i}\right\}$ of $\left\{\hat{E}_{\alpha}\right\}$ such that $\bigcup_{i=1}^{\infty} \hat{E}_{i}=\hat{X}$ a.e. Let $m_{1}=\sup \left\{\mu \hat{E}_{\alpha}: \hat{E}_{\alpha} \in M\right\}$. Take a set $\hat{E}_{1}$ such that $\mu \hat{E}_{1}>m_{1} / 2$. Take a set $\hat{E}_{i}$ successively for $i=2,3, \ldots$ such that $\mu \hat{E}_{i}>m_{i} / 2$ where $m_{i}=\sup \left\{\mu \hat{E}_{\alpha}: \hat{E}_{\alpha} \in \mathcal{M}, \hat{E}_{\alpha} \subset \hat{X}-\bigcup_{k=1}^{i-1} \hat{E}_{k}\right\}$. Using a standard argument, it is easy to show that the union of $\hat{E}_{i}$ 's is $\hat{X}$ a.e. Let $E_{i}=\pi\left(\hat{E}_{i}\right)$ where $\pi$ is the map 
identifying $x$ and $S(x)$. Clearly the collection $\left\{E_{i}\right\}$ satisfies definition 2 . This completes the proof.

If an ergodic foliation arises from an ergodic action, then it is clear from the construction of $T$ that the covering space has two ergodic components (To define $T$ on $X_{1}$, we do not need $X_{2}$ ). Also it is clear that if we can put an ergodic action on a foliation by changing the direction of a measurable subset, then the covering space will have two ergodic components. We may note that there is a natural map $\pi:(\hat{X},\{T, S\}, \hat{\mu}, \hat{F}) \rightarrow(X, \mathscr{E}, \mu, \mathscr{F})$ such that $\pi\left(x_{1}\right)=\pi S\left(x_{1}\right)=\pi\left(x_{2}\right)=x$. In this sense, an ergodic foliation may be regarded as a generalization of a factor (a quotient space).

One of the concrete examples of an ergodic foliation comes from the adding machine. Let $(\hat{X}, \hat{\mu}, \hat{F})$ be a product space $\Pi(\{0,1\}, p\}$ where $p(0)=p(1)=\frac{1}{2}$. Define the transformation $T$ by

$$
(T x)_{n}= \begin{cases}0 & \text { if } n<I(x) \\ 1 & \text { if } n=I(x) \\ x_{n} & \text { if } n>I(x)\end{cases}
$$

where $I(x)=\min \left\{i \in \mathbb{N}: x_{i}=0\right\}$.

Let $S$ be the interchange map between 0 's and 1's. Clearly $S$ is an order two map. It is easy to check that $S$ skew commutes with $T$. Let $X$ be a quotient space by identifying $x$ and $S(x)$. Clearly $(\hat{X},\{T, S\}, \hat{\mu}, \hat{\mathscr{F}})$ is a covering space of the ergodic foliation $(X, \mathscr{E}, \mu, \mathscr{F})$ where $\mathscr{F}$ is the quotient $\sigma$-algebra.

\section{Properties of a measurable ergodic foliation}

The following theorem will enable us to lift problems of a foliation to those of a covering space.

THEOREM 3. Two foliations $\left(X_{1}, \mathscr{E}_{1}, \mu_{1}, \mathscr{F}_{1}\right)$ and $\left(X_{2}, \mathscr{E}_{2}, \mu_{2}, \mathscr{F}_{2}\right)$ are isometric if and only if their covering spaces $\left(\hat{X}_{1},\left\{T_{1}, S_{1}\right\}, \hat{\mu}_{1}, \hat{\mathscr{F}}_{1}\right)$ and $\left(\hat{X}_{2},\left\{T_{2}, S_{2}\right\}, \hat{\mu}_{2}, \hat{\mathscr{F}}_{2}\right)$ are isomorphic.

Proof. $(\Rightarrow)$ Let $\varphi$ be an isometry between two foliations. We want to define an isomorphism $\hat{\varphi}$ on $\hat{X}_{1}$. Let $\hat{x}$ be a point in $\hat{X}_{1}$. Let $\pi_{1}(\hat{x})=x$ and $\varphi(x)=y$. Let $\hat{y}$ and $\hat{y}^{\prime}$ be two points in $\hat{X}_{2}$ satisfying $\pi_{2}(\hat{y})=\pi_{2}\left(\hat{y}^{\prime}\right)=y$. We define $\hat{\varphi}(\hat{x})=\hat{y}$ where $\varphi \pi_{1}\left(T_{1} \hat{x}\right)=\pi_{2}\left(T_{2} \hat{y}\right)$. Since $\varphi$ is an isometry, $\varphi \pi_{1}\left(T_{1} \hat{x}\right)$ is either $\pi_{2}\left(T_{2} \hat{y}\right)$ or $\pi_{2}\left(T_{2} \hat{y}^{\prime}\right)$. It is not hard to see that $\hat{\varphi}$ is one-to-one and measurable. By the definition of $\hat{\varphi}$, we have

(i) $\pi_{2} \hat{\varphi}(\hat{x})=\pi_{2}(\hat{y})=y$

(ii) $\varphi \pi_{1}\left(T_{1} S_{1} \hat{x}\right)=\varphi \pi_{1}\left(S_{1} T_{1}^{-1} \hat{x}\right)=\varphi \pi_{1}\left(T_{1}^{-1} \hat{x}\right)=\pi_{2}\left(T_{2}^{-1} \hat{y}\right)=\pi_{2} S_{2}\left(T_{2}^{-1} \hat{y}\right)$ $=\pi_{2} T_{2}\left(S_{2} \hat{y}\right)$

Hence $\hat{\varphi}\left(\hat{x}^{\prime}\right)=\hat{\varphi}\left(S_{1} \hat{x}\right)=S_{2} \hat{y}=\hat{y}^{\prime}$. Therefore, $\hat{\varphi}\left(S_{1} \hat{x}\right)=S_{2} \hat{\varphi}(\hat{x})$. Next we want to show $\hat{\varphi} \cdot T_{1}=T_{2} \cdot \hat{\varphi}$. By the definition of $\hat{\varphi}$, we know $\hat{\varphi}\left(T_{1} \hat{x}\right)$ is either $T_{2} \hat{\varphi}(\hat{x})$ or $S T_{2} \hat{\varphi}(\hat{x})$. If $\hat{\varphi}\left(T_{1} \hat{x}\right)=S_{2} T_{2} \hat{\varphi}(\hat{x})$, then we have

$$
\varphi \pi_{1}\left(T_{1}^{2} \hat{x}\right)=\pi_{2}\left(T_{2} S_{2} T_{2} \hat{\varphi}(\hat{x})\right)=\pi_{2}\left(T_{2} T_{2}^{-1} S_{2} \hat{\varphi}(\hat{x})\right)=\pi_{2}\left(S_{2} \hat{\varphi}(\hat{x})\right)=\pi_{2} \hat{\varphi}(\hat{x}) .
$$


Therefore,

$$
d\left(\pi_{1} \hat{x}, \pi_{1} T_{1}^{2} \hat{x}\right)=d\left(\varphi\left(\pi_{1} \hat{x}\right), \varphi\left(\pi_{1} T_{1}^{2} \hat{x}\right)\right)=d\left(\pi_{2} \hat{\varphi}(\hat{x}), \pi_{2} \hat{\varphi}(\hat{x})\right)=0 .
$$

This is a contradiction since $d\left(\pi_{1} \hat{x}, \pi_{1} T_{1}^{2} \hat{x}\right)=d\left(\hat{x}, T_{1}^{2} \hat{x}\right)=2$. Hence $\hat{\varphi}\left(T_{1} \hat{x}\right)=T_{2} \hat{\varphi}(\hat{x})$.

$(\Leftarrow)$ Let $x$ be a point in $\left(X_{1}, \mathscr{E}_{1}, \mu_{1}, \mathscr{F}_{1}\right)$. If two covering spaces are isomorphic via $\hat{\varphi}$, then we define $\varphi(x)=\pi_{2} \hat{\varphi}(\hat{x})$ where $\hat{x}$ is one of the points in the covering space corresponding to $x$. Since $\pi_{2} \hat{\varphi}(\hat{x})=\pi_{2} S \hat{\varphi}(\hat{x})=\pi_{2} \hat{\varphi} S(\hat{x})=\pi_{2} \hat{\varphi}\left(\hat{x}^{\prime}\right)$ where $\hat{x}^{\prime}$ is the other point in $X_{1}$ corresponding to $x, \varphi(x)$ is well defined and clearly measurable. Also $\varphi$ is leaf preserving since $\hat{\varphi}$ is orbit preserving. We let $x$ and $y$ be two points in a leaf in $X_{1}$ and $\hat{x}$ and $\hat{y}$ be the points in $\hat{X}_{1}$ corresponding to $x$ and $y$, respectively, where $\hat{x}$ and $\hat{y}$ are in the same orbit under $T_{1}$. Then

$$
d(\varphi(x), \varphi(y))=d(\pi \hat{\varphi}(\hat{x}), \pi \hat{\varphi}(\hat{y}))=d(\hat{\varphi}(\hat{x}), \hat{\varphi}(\hat{y}))=d(\hat{x}, \hat{y})=d(x, y) .
$$

Hence $\varphi$ is an isometry.

We denote $L^{(n)}(x)=\left\{x=x^{0}, x^{1}, x^{2}, \ldots, x^{n}\right\}$ to be the set of points in the leaf of $x$ where the distance between two successive points is 1 .

Corollary 1. Let $E$ be a subset of positive measure.

$$
\lim _{n \rightarrow \infty} \frac{\#\left\{L^{n}(x) \cap E\right\}}{n+1}=\mu E \text { a.e. }
$$

Proof. If the foliation comes from an ergodic action, then the corollary is clear. Otherwise, we may assume that $T$ in the covering space is ergodic. Let $\hat{x}$ be the point in the covering space such that $\pi\left(T^{i} \hat{x}\right)=x^{i}$ for $i=0,1, \ldots$ Let $E=\hat{E}_{1} \cup \hat{E}_{2}$ where $\pi\left(\hat{E}_{1}\right)=\pi\left(\hat{E}_{2}\right)=E$. By the ergodicity of $T$, we have

$$
\lim _{n \rightarrow \infty} \frac{\#\left\{\left\{T^{i} \hat{x}\right\}_{i=0}^{n} \cap \hat{E}\right\}}{n+1}=\frac{\hat{\mu} \hat{E}}{\hat{\mu} \hat{x}}=\mu E .
$$

Since we have $\#\left\{L^{n}(x) \cap E\right\}=\#\left\{\left\{T^{i} \hat{x}\right\}_{i=0}^{n} \cap \hat{E}\right\}$, the corollary follows.

Let $P=\left\{P_{0}, P_{1}, \ldots\right\}$ be a partition of a foliation $(X, \mathscr{E}, \mu, \mathscr{F})$. We define a partition $\hat{P}=\left\{\hat{P}_{0}, \hat{P}_{0}^{\prime}, \hat{P}_{1}, \hat{P}_{1}^{\prime}, \ldots\right\}$ of a covering space $\hat{X}=X_{1} \cup X_{2}$ such that $\hat{P}_{i} \subset X_{1}, \hat{P}_{i}^{\prime} \subset X_{2}$ and $\pi\left(\hat{P}_{i}\right)=\pi\left(\hat{P}_{i}^{\prime}\right)=P_{i}$. We say a partition $P$ generates the $\sigma$-algebra $\mathscr{F}$ of $X$ under the foliation if two different points have two different leaf names of $P$. Leaf names are like orbit names without direction. If $P$ generates the $\sigma$-algebra of $X$ under the foliation, then it is clear that $\hat{P}$ generates the $\sigma$-algebra $\hat{\mathscr{F}}$ under $T$.

Corollary 2. Let $P=\left\{P_{0}, \ldots, P_{k}\right\}$ be a partition of a foliation $(X, \mathscr{E}, \mu, \mathscr{F})$. For a given $n$ and $\varepsilon>0$, there exists a Rokhlin tower of height $\geq n$ such that every point in the base satisfies the ergodic theorem for a foliation for each set of the partition within $\varepsilon$.

Proof. Since we have Lemma 2 and Corollary 1, the proof is analogous to that of an ergodic $\mathbb{Z}$-action.

Earlier we defined leaf-equivalence between two ergodic foliation. Now we are ready to prove the equivalence theorem, whose proof is based on Corollary 2 .

THEOREM 4. Any two ergodic foliations are leaf-equivalent. 
Proof. Let $\left(X_{1}, \mathscr{C}_{1}, \mu_{1}, \mathscr{F}_{1}\right)$ and $\left(X_{2}, \mathscr{C}_{2}, \mu_{2}, \mathscr{F}_{2}\right)$ be two ergodic foliations. Let $\left\{P^{(i)}\right\}$ be a sequence of finite partitions such that

(i) $P^{(i+1)}$ is a refinement of $P^{(i)}$.

(ii) $\bigvee_{i=1}^{\infty} P^{(i)}$ generates $\mathscr{F}$ under the foliation.

We may assume that there exists a finite partition $Q=\left\{Q_{1}, \ldots, Q_{k}\right\}$ of $X_{2}$ which generates the $\sigma$-algebra under the foliation. We will define an equivalence map $\varphi$ as a limit of successive set maps $\left\{\varphi^{(i)}\right\}$. Let $\sum \varepsilon_{i}<\infty$.

First, we define $\varphi^{(1)}$. We construct a Rokhlin tower of height $n_{1}$ of $X_{1}$ such that

(i) The error set has measure less than $\varepsilon_{1}$.

(ii) Each point of the base satisfies the ergodic theorem for every set in $P^{(1)}$ within $\varepsilon_{1}$. If Rokhlin tower satisfies both (i) and (ii) with respect to the partition $P$, then we say that the Rokhlin tower has property $R\left(P, \varepsilon_{1}\right)$. Construct a Rokhlin tower of $X_{2}$ of height $n_{1}$ such that it has the property $R\left(Q, \varepsilon_{1}\right)$. We may assume that both Rokhlin towers have the same measure. In other words, the bases of two towers, denoted by $F^{(1)}$ and $E^{(1)}$, respectively, have the same measure. We partition the bases of both towers according to $P^{(1)}$ and $Q$ names, respectively, and subpartition the bases into $F_{j}^{(1)}$ 's and $E_{j}^{(1)}$ 's such that

(i) each of $F_{j}^{(1)}$ 's and $E_{j}^{(1)}$ 's has n.le $P$-name or $Q$-name respectively,

(ii) $F^{(1)}=\bigcup_{j=1}^{t_{1}} F_{j}^{(1)}$ and $E^{(1)}=\bigcup_{j=1}^{t_{1}} E_{j}^{(1)}$.

(iii) $\mu_{1} F_{j}^{(1)}=\mu_{2} E_{j}^{(1)}$ for $j=1, \ldots, t_{1}$.

We denote the $k$ th level set above $F_{j}^{(1)}$ by $F_{j, k}^{(1)}$ for $k=0,1, \ldots, n_{1}-1$. Define a set map $\varphi^{(1)}$ on this Rokhlin tower so that $\varphi^{(1)}\left(F_{j, k}^{(1)}\right)=E_{j, k}^{(1)}$ for $j=1, \ldots, t_{1}$ and $k=$ $0, \ldots, n_{1}-1$. We note that $\varphi^{(1)}$ is defined except on the error set.

Now we define $\varphi^{(i+1)}$ from $\varphi^{(i)}$. Let

$$
\eta^{(i)}=\min \left\{\mu_{1} F_{1}^{i}, \mu_{1} F_{2}^{i}, \ldots, \mu_{i} F_{t i}^{i}, \mu_{2} E_{1}^{i}, \ldots, \mu_{2} E_{t i}^{i}\right\} .
$$

We take Rokhlin towers of height $n_{i+1}$ in both spaces such that

(i) $n_{i} / n_{i+1}<\varepsilon_{i+1} / 10$

(ii) Error set has measure $<\varepsilon_{i+1} / 10$

(iii) Every point in the base $F^{(i+1)}$ satisfies the ergodic theorem within $\left(\varepsilon_{i+1} / 10\right) \cdot \eta^{(i)}$ for $F_{j}^{(i)}\left(j=1, \ldots, t_{i}\right)$

(iv) Every point in the base $E^{(i+1)}$ satisfies the ergodic theorem within $\left(\varepsilon_{i+1} / 10\right) \cdot \eta^{(i)}$ for $E_{j}^{(i)}\left(j=1, \ldots, t_{i}\right)$.

We partition $F^{(i+1)}$ and $E^{(i+1)}$ according to $P^{(i+1)}$-names and $Q$-names respectively. Subpartition each atom of the partition so that

(i) $\bigcup_{j=1}^{i_{i+1}} F_{j}^{(i+1)}=F^{(i+1)}$ and $\bigcup_{j=1}^{t_{i+1}} E_{j}^{(i+1)}=E^{(i+1)}$

(ii) $\mu_{1} F_{j}^{(i+1)}=\mu_{2} E_{j}^{(i+1)}$ for all $j=1, \ldots, t_{i+1}$.

We define $\varphi^{(i+1)}$ in detail on the first column of the Rokhlin tower, whose base is $F_{1}^{(i+1)} \cdot \varphi^{(i+1)}$ is defined analogously on the other columns of the tower. Let $E$ be a subset of the base of the $i$ th Rokhlin tower and $E^{k}$ be the $k$ th level set above $E$. We call the set $\bigcup_{k=0}^{n_{i}-1} E^{k}$ a subcolumn of the $i$ th tower. $\varphi^{(i+1)}$ maps the first column starting with $F_{1}^{(i+1)}$ to the first column starting with $E_{1}^{(i+1)}$. Let $F_{i, k_{1}}^{(i+1)}$ be the first level set in the column such that $F_{1, k_{1}}^{(i+1)}$ is contained in $F_{j_{1}}^{(i)}$ for some $j_{1}$. That is, $F_{1, k_{1}}^{(i+1)}$ is the first level set in the column where a subcolumn of the $i$ th Rokhlin tower 
starts. (The subcolumn of the $i$ th Rokhlin tower starting $F_{1, k_{1}}^{(i+1)}$ could be either upward or downward unlike the case of an ergodic action.) Let $E_{1, l_{1}}^{(i+1)}$ be the first level set in the first column of the Rokhlin tower such that $E_{1, l_{1}}^{(i+1)}$ is contained in $E_{j_{1}}^{(i)}$. We call the subcolumn starting with $E_{1, l_{1}}^{(i+1)}$ the corresponding subcolumn to the subcolumn starting with $F_{1, k_{1}}^{(i+1)}$. We define $\varphi^{(i+1)}$ to map the subcolumn of the $i$ th Rokhlin tower starting with $F_{1, k_{1}}^{(i+1)}$ to the subcolumn of the $i$ th Rokhlin tower of $X_{2}$ starting with $E_{1, l_{1}}^{(i+1)}$. We define $\varphi^{(i+1)}$ on this section of the column so that $\varphi^{(i+1)}$ is consistent with $\varphi^{(i)}$ on this subcolumn of the $i$ th Rokhlin tower. We recall that $\varphi^{(i)}$ maps the $i$ th Rokhlin tower starting with $F_{j}^{(i)}$ to the $i$ th Rokhlin tower starting with $E_{j}^{(i)}$ for $j=1,2, \ldots, t_{i}$. If the second subcolumn of the $i$ th Rokhlin tower starts with $F_{1, k_{2}}^{(i+1)}$, which is a subset of $F_{j_{2}}^{(i)}\left(j_{1} j_{2}\right)$, then we define $\varphi^{(i+1)}$ to map this section of the tower to the subcolumn of the $i$ th Rokhlin tower starting with $E_{1, l_{2}}^{(i+1)}$, which is the first level set contained in $E_{j_{2}}^{(i)}$. Again we require $\varphi^{(i+1)}$ to be consistent with $\varphi^{(i)}$ on this subcolumn of the ith Rokhlin tower. If $F_{1, k_{2}}^{(i+1)} \subset F_{j_{1}}^{(i)}$, then we define $\varphi^{(i+1)}$ to map this section of the tower to the subcolumn of the $i$ th Rokhlin tower starting with $E_{1, l_{2}}^{(i+1)}$, which is the first level set above $l_{1}$ contained in $E_{j_{1}}^{(i)}$ in the column.

Similarly we define $\varphi^{(i+1)}$ on each of the subcolumns of the $i$ th Rokhlin towers appearing in the first column as long as it has a corresponding subcolumn of the $i$ th Rokhlin tower of $X_{2}$. If a subcolumn does not have a corresponding subcolumn in the first column of the $(i+1)$ st Rokhlin tower of $X_{2}$, then we go up to the next subcolumn of the $i$ th Rokhlin tower to define $\varphi^{(i+1)}$. By the ergodic theorem, there are at most $2 \cdot\left(\varepsilon_{i+1} / 10\right) \cdot \mu F^{(i)} \cdot n_{i+1}=\left(\varepsilon_{i} / 5\right) \cdot \mu F^{(i)}$ many subcolumns of the $i$ th Rokhlin tower where $\varphi^{(i+1)}$ cannot be defined so far. We may assume that in each column of the $(i+1)$ st Rokhlin tower there are $\left(\varepsilon_{i}+2 \varepsilon_{i+1}\right) \cdot n_{i+1}$-many level sets contained in the error set of the $i$ th Rokhlin tower. Let $\mathscr{L}$ be a collection of the

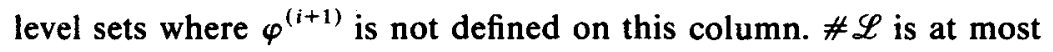

$$
\left(\varepsilon_{i}+2 \varepsilon_{i+1}\right) \cdot n_{i+1}+\left(\varepsilon_{i+1} / 5\right) \cdot \mu F^{(i)} \cdot n_{i+1} \cdot n_{i} \leq\left(\varepsilon_{i}+2 \varepsilon_{i+1}+\varepsilon_{i+1} / 5\right) \cdot n_{i+1}
$$

since $\mu F^{(i)} \cdot n_{i}<1$.

On these level sets in $\mathscr{L}$, we define $\varphi^{(i+1)}$ linearly. That is, the $k$ th level set in $\mathscr{L}$ is mapped to the $k$ th level set in $\mathscr{L}^{\prime}$, a collection of the level sets in the first column of the $(i+1)$ st Rokhlin tower of $X_{2}$ which are not contained in the image of $\varphi^{(i+1)}$ so far. Since two Rokhlin towers have the same height at every stage, $\varphi^{(i+1)}$ is a one-to-one (not pointwise, but level by level) and onto map on the first column of $X_{1}$ to the first column of $X_{2}$. We extend the definition of $\varphi^{(i+1)}$ to the whole $(i+1)$ st Rokhlin tower.

We want to compute the measure of the set $D_{i+1}$ where $\varphi_{i}$ and $\varphi_{i+1}$ differ. Clearly $\mu D_{i+1} \leq \mu C_{i}+\mu C_{i+1}+\left(\varepsilon_{i+1} / s\right)<\varepsilon_{i}$ where $C_{i}$ is the error set of the $i$ th Rokhlin tower. We note that $\varepsilon_{i+1} / 5$ is the measure of the subset of the $i$ th Rokhlin tower where $\varphi^{(i+1)}$ is defined differently from $\varphi^{(i)}$. Since $\sum \varepsilon_{i}<\infty$, by Borel-Cantelli Lemma, for a.e. $x$ there exists $n(x)$ such that $x \in D_{i}^{c}$ for all $i \geq n(x)$. Hence we define $\varphi=$ $\lim _{i \rightarrow \infty} \varphi^{(i+1)}$. Clearly $\varphi$ is measure preserving and leaf preserving. This completes the proof. 
Since we have this weak isomorphism (leaf equivalence), Kakutani equivalence and isometry to characterize foliations, one of the interesting areas to be pursued further would be to investigate what the role of D. Rudolph's restricted orbit equivalence theory might be in this larger class of ergodic foliations (larger than the class of ergodic actions) [10].

\section{Part II. Construction of a counterexample}

In Part I, we showed that problems of an isometry between foliations may be lifted to those of isomorphism between two group actions in the covering space. If two covering spaces have non-isomorphic $\mathbb{Z}$-actions, then they give rise to non-isometric foliations. Let $G_{i}$ be a group generated by two transformations, $T_{i}$ and $S_{i}$ where $S_{i}^{2}=I$ and $S_{i} T_{i} S_{i}=T_{i}^{-1}$ for $i=1$ and 2 . It is known that if $\left(\hat{X}_{i}, T_{i}, \hat{\mu}_{i}, \hat{\mathscr{F}}_{i}\right)$ are Bernoulli with the same entropy (that is, the $T_{i}$ 's are isomorphic), then an isomorphism between two $\mathbb{Z}$-actions forces an isomorphism between $\left(\hat{X}_{1}, G_{1}, \hat{\mu}_{1}, \hat{\mathscr{F}}_{1}\right)$ and $\left(\hat{X}_{2}, G_{2}, \hat{\mu}_{2}, \hat{F}_{2}\right)$ [4]. On the other hand, if $T_{1}$ is a rotation of a circle, it is well known that $T_{1}$ is isomorphic only to itself or its inverse via another rotation. Therefore if $T_{1}$ and $T_{2}$ are irrational rotations on the unit circle and isomorphic (that is $T_{2}=T_{1}$ or $\left.T_{2}=T_{1}^{-1}\right)$, then $\left(\hat{X}_{1}, G_{1}, \hat{\mu}_{1}, \hat{\mathscr{F}}_{1}\right)$ and $\left(\hat{X}_{2}, G_{2}, \hat{\mu}_{2}, \hat{\mathscr{F}}_{2}\right)$ are isomorphic. This is true because any map of order two which skew-commutes with a rotation is a 'folding' map. By a folding map $S$, we mean a map satisfying $S\left(e^{2 \pi i t}\right)=e^{2 \pi i(2 \theta-t)}$ for some $\theta$. If we have $\left\{T, S_{1}\right\}$ and $\left\{T^{-1}, S_{2}\right\}$ where $S_{1}$ and $S_{2}$ are different folding maps by $\theta_{1}$ and $\theta_{2}$, respectively, then we define $\varphi$ such that $\varphi\left(e^{2 \pi i t}\right)=e^{2 \pi i\left(\theta_{1}+\theta_{2}-t\right)}$, which is another folding map by $\left(\theta_{1}+\theta_{2}\right) / 2$. It is easy to show that $\varphi$ is an isomorphism between $\left\{T, S_{1}\right\}$ and $\left\{T^{-1}, S_{2}\right\}$. Hence they give isometric foliations. If we have $\left\{T, S_{1}\right\}$ and $\left\{T, S_{2}\right\}$, then it is also easy to see that $\varphi\left(e^{2 \pi i t}\right)=e^{2 \pi i\left(\theta_{1}-\theta_{2}+\imath\right)}$ is an isomorphism.

We want to construct a $K$-automorphism which gives two non-isometric foliations; that is, a $K$-automorphism which admits two order two maps, $S_{1}$ and $S_{2}$, giving rise to two non-isomorphic bigger group actions. One of the interesting questions along this line is whether this property is true for other kinds of mixing $\mathbb{Z}$-actions. We mention that D. Rudolph has shown that if the $T_{i}$ 's are Bernoulli and the $S_{i}$ 's commute with the $T_{i}$ 's respectively, then an isomorphism between the $T_{i}$ 's forces an isomorphism of bigger groups [9]. A counterexample has been constructed to show that this property does not hold for $K$-automorphisms [6].

\section{Construction of $(\hat{X},\{T, S\}, \hat{\mathscr{F}}, \hat{\mu})$.}

Outline of the construction is as follows. We first construct a $K$-automorphism which is similar to the one of D. Ornstein [3]. We make a two point extension by adding colours following D. Rudolph's construction [8]. Hence every point in the space is specified by a pair of names, called a base name and a colored name. We define two order two maps, $S_{1}$ and $S_{2}$, in such a way that both of them skew-commute with the $K$-automorphism, but they give rise to non-isomorphic bigger group actions. This $K$-automorphism $T$ is isomorphic to its inverse via order two maps. The two maps we are going to construct are 'flips' between the left and the right of the 
coordinates, one of only a base name and the other of a double name. Hence we need sufficient "symmetry" in the construction of the $K$-automorphism so that these two flip maps are well defined. We will show by using the rigidity of blocks and the regularity of coloured names that there is no isomorphism between $\left\{T, S_{1}\right\}$ and $\left\{T, S_{2}\right\}$.

First we will construct a transformation $U$, a version of Ornstein's $K$ automorphism. We refer the reader to [5] for more geometric and rigorous construction. As we construct the transformation $U$, at each stage we construct a set map $S^{(i)}$ in such a way that $S^{(i)}$ is defined on a bigger set than $S^{(i-1)}$ and $S^{(i)}$ is a refinement of $S^{(i-1)}$ where $S^{(i-1)}$ is defined. We will use the limit of these $S^{(i)}$ s to construct $S_{1}$ and $S_{2}$ after we construct the $K$-automorphism $T$.

The transformation $U$ will be defined as a shift on a space $X$ of bilateral sequences of three symbols $\{o, f, s\}$ relative to a measure on $X$. The construction will show how to determine the measure. Each sequence $x$ in $X$ is made up of nested blocks, called $n$-blocks. Each $n$-block has length $h(n)$, which is specified as we define an $n$-block. Each $n$-block is made up of $(n-1)$-blocks, spacers ( $s$ 's) and $f$ 's. We define one 1-block name which begins with two $s$ 's followed by $2^{100}$ consecutive $o$ 's, and finally by two $s$ 's again. We define $S^{(1)}$ as a set map on this 1-block. Let $B$ be the level set where this 1-block starts. We define $S^{(1)}\left(U^{i} B\right)=U^{h(1)-i-1} B$ for $i=0$, $1, \ldots, h(1)-1$.

Construction of an $n$-block name from $(n-1)$-block names goes as follows. Choose independently a sequence of $\left(2^{2 n}+1\right)(n-1)$-block names. Let $f(n)$ be a random integer out of the set $\{1,2, \ldots, n\}$, (we want $(n+1)-f(n) \in\{1,2, \ldots, n\}$ to be equidistributed) independent of the chosen sequence of $(n-1)$-blocks. We construct two $n$-block names out of these choices. Each of them begins with a string of $f \mathrm{~s}, f(n)$ long, and ends with a string of $f \mathrm{~s}, n+1-f(n)$ long. In between, one of these has the chosen $\left(2^{2 n}+1\right)(n-1)$-block names separated by strings of spacers in increasing order. That is, the length of spacers between the $k$ th $(n-1)$-block and the $(k+1)$ st $(n-1)$-block is $k s(n)$. $(s(n)$ will be defined later.) The other has the chosen $\left(2^{2 n}+1\right)(n-1)$-block names separated by strings of spacers in decreasing order (see figure 3 ).

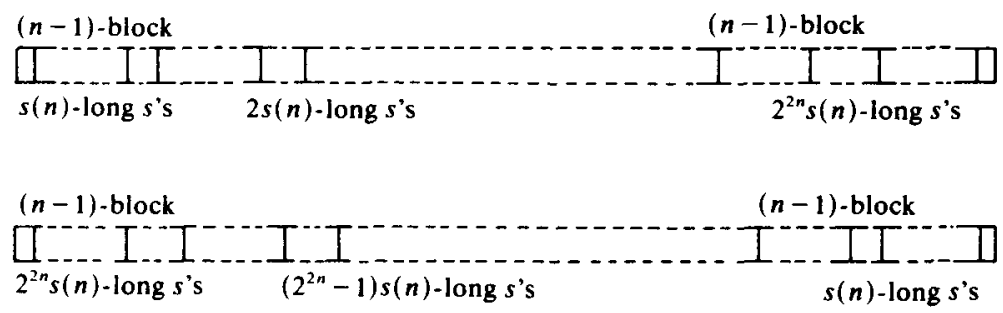

FIGURE 3. Two $n$-blocks with the same $\left(2^{2 n}+1\right)(n-1)$-blocks and $f(n)$.

This gives the various $n$-block names, each with equal probability. The number of $n$-block names is $k(n)=2(n)(k(n-1))^{2^{2 n}+1}$. We note that for each $n$-block name $A_{n, s}(s=1,2, \ldots, k(n))$ there is an $n$-block name, $A_{n, t}$ of the same probability which 
is the same except in a complete reverse order. We call these $\boldsymbol{n}$-block names $\boldsymbol{A}_{n, t}$ an echo block name of $A_{n, s}$. To make our understanding of $S^{(n)}$ clear, we will explicitly define $S^{(2)}$ on 2-blocks. We note that for each $f(2) \in\{1,2\}$, there are two different 2-block names according to the way the spacers are put in. Hence there are four 2-block names. Also each of these four names has a name in a complete reverse order. We assume that the 2-block names $A_{2,1}$ and $A_{2,2}$ are in reverse order. Let $\left\{B_{2, i}\right\}_{i=1}^{4}$ be the level sets where these 2-blocks start. We define $S^{(2)}\left(U^{i} B_{2,1}\right)=$ $U^{h(2)-1-i} B_{2,2}$ and $S^{(2)}\left(U^{i} B_{2,2}\right)=U^{h(2)-1-i} B_{2,1}$ for $i=0,1, \ldots, h(2)-1$. We also define $S^{(2)}$ on the other pair of 2-blocks in an analogous way. It is clear that the definition of $S^{(2)}$ on 2-blocks is consistent with $S^{(1)}$ on the 1-block. Let $B_{n, s}$ $(s=1, \ldots, k(n))$ be the level set where the $s$ th $n$-block starts and $B_{n, t}$ be the level set where the echo $n$-block of $A n, s$ starts. We define $S^{(n)}$ on this $n$-block by $S^{(n)}\left(U^{i} B_{n, s}\right)=U^{h(n)-1-i}\left(B_{n, t}\right)$ for $i=0, \ldots, h(n)-1$. It is not hard to see that the definition of $S^{(n)}$ on $(n-1)$-blocks is consistent with $S^{(n-1)}$ on $(n-1)$-blocks. Also $S^{(n)}$ satisfies the following:

(i) $S^{(n)^{2}}=I$

(ii) $\operatorname{SUS}\left(U^{i} B_{n, s}\right)=S U\left(U^{h(n)-1-i} B_{n, t}\right)$

$$
\begin{aligned}
& =S\left(U^{h(n)-i} B_{n, t}\right)=U^{h(n)-1-(h(n)-i)} B_{n, s}=U^{i-1} B_{n, s} \\
& =U^{-1}\left(U^{i} B_{n, s}\right), \text { for } i=1,2, \ldots, h(n)-1 .
\end{aligned}
$$

That is, $S U S=U^{-1}$ on each level set of an $n$-block.

If we let $s(n)=100 n^{3}(n=2,3, \ldots)$, then certainly $s(n+1)>100 \sum_{i=1}^{n} f(i)$. Also since we have chosen $h(1)$ to be long enough, it is not hard to see that the total measure of $s$ 's and $f$ 's added in going from $X_{n-1}$ (the set of all $(n-1)$-blocks) to $X_{n}$ is bounded by $\mu\left(X_{n}\right) / 2^{6 n}$. Hence the $\mu\left(X_{n}\right)$ 's are uniformly bounded.

We note here that an $(n-1)$-block occurs at all possible $(n-1)$-block places in an $n$-block with equal probability, independent of each other. And any $n$-block has its echo $n$-block of the same probability. If we call $X$ the space where $U$ is well defined, then we have a partition on $X$ according to the symbols used, $P=$ $\left\{P_{o}, P_{f}, P_{s}\right\}$. Since the level sets of $n$-blocks $(n=1,2, \ldots)$ generate the $\sigma$-algebra, there is a well defined measurable map $S: X \rightarrow X$ such that if $\left\{x_{i}\right\}$ is a name of $x$, then $S(x)=x^{\prime}$ where $x^{\prime}=\left\{x_{i}^{\prime}\right\}=\left\{x_{-i}\right\}$. That is, $S$ is a 'flip' map between left and right with respect to $x_{0}$. This map is clearly of order two and it satisfies $S U S(x)=$ $S U\left(\left\{x_{-i}\right\}\right)=S\left(\left\{x_{-i+1}\right\}\right)=\left\{x_{i-1}\right\}=U^{-1}(x)$. The existence of this map $S$ on $X$ will enable us to construct two different such automorphisms on a bigger space to be constructed.

To construct the $K$-automorphism $T$, let $\hat{X}$ be the direct product of $X$ with a two point space $\{B, W\}$. We partition $\hat{X}$ into $\left\{P_{o} \times\{W\}, P_{o} \times\{B\}, P_{f} \times\{B, W\}\right.$, $\left.P_{s} \times\{B, W\}\right\}=\left\{W_{o}, B_{o}, P_{s}, P_{f}\right\}$. We call this partition $\bar{P}$. We will say that a point is in $W$ if it is in $W_{o}$ and in $B$ if it is in $B_{o}$ as coloured white or black. We will define $T$ for a point in $\hat{X}$ by specifying changes of colours at each $n$-block. For each $n$, there are two different coloured $n$-block names whose base names are the same. We will describe how to colour an $n$-block from coloured $(n-1)$-blocks. We call an $(n-1)$-block white (black) if its colour starts with white (black). Construction 
of a white $n$-block goes as follows. Choose a sequence of $2^{2 n}+1(n-1)$-blocks independently according to base names. Choose the first $(n-1)$-block to be white, second to be black, third to be black, fourth to be white and fifth to be white, ..., so that the colours of $(n-1)$-blocks in a white $n$-block is $W B B W W B B W W B B \cdots W W$. A black $n$-block is an $n$-block with the configuration of colours, $B W W B B \cdots B B$. With the same configuration of colours and the sequence of $(n-1)$-blocks, we have two $n$-blocks of equal probability, one with the spacers in increasing order and the other with them in decreasing order.

We may regard this $T$ as a skew product of $U$ with an interchange map between white and black. We want to define two different automorphisms of order two, $S_{1}$ and $S_{2}$. We define

$$
\begin{aligned}
& S_{1}(x, w)=\left(x^{\prime}, w\right), \quad w=W \text { or } B \text { and } x^{\prime}=\left\{x_{i}^{\prime}\right\}=\left\{x_{-i}\right\} \text { for } i=0, \pm 1, \pm 2, \ldots \\
& S_{2}(x, w)= \begin{cases}\left(x^{\prime}, B\right) & \text { if } w=W \\
\left(x^{\prime}, W\right) & \text { if } w=B .\end{cases}
\end{aligned}
$$

It is clear that $S_{1}^{2}=I$ and $S_{i} T S_{i}=T^{-1}$ for $i=1$ and 2 .

\section{2. $T$ is a K-automorphism}

Since the proof in this section is parallel to that in [5] and [8], we will just sketch the proof for the completeness of the paper. We note two major differences in the construction of $U$ from the general construction of a $K$-automorphism.

(1) We put $(n+1)-f(n) f$ 's at the ends of $n$-blocks instead of $e$ 's.

(2) With given $f(n)$ and $2^{2 n}+1(n-1)$-block names, we construct two $n$-blocks of equal probability, one in the increasing order of spacers and the other in the decreasing order. But from the construction, it is not hard to see that these changes do not affect the rigidity of blocks and the randomness of the occurences of $(n-1)$-blocks in the possible positions in their $n$-blocks, hence in bigger blocks. We want to show that the distant past has little effect on its future. We will use the following definition of a $K$-automorphism. For given $l$ and $\varepsilon>0$, there is an $N$ such that $\bigvee_{-1}^{o} T^{i} \bar{P}$ is $\varepsilon$-independent of $\bigvee_{n}^{n+k} T^{i} \bar{P}$ for any $k$ and any $n>N$.

\section{THEOREM 5. $T$ is a $K$-automorphism}

Proof. We fix $k$ and choose $K$ such that for most $x \in \hat{X},\left\{x, T x, T^{2} x, \ldots, T^{k} x\right\}$ are completely in a $K$-block. It is clear that for most $x \in \hat{X},\left\{T^{n} x, \ldots, T^{n+k} x\right\}$ are completely contained in a $K$-block. Let $x$ be a point satisfying this condition which is in one of the atoms $A$ of $\bigvee_{-l}^{o} T^{i} \bar{P}$. We choose $L$ so large that $L \gg h(K+1)$. We let $N=h(L)+1$ and $n>N$. Since $n>N=h(L)+1$, and an $L$-block starts somewhere between $x$ and $T^{n} x$. We note the following:

(1) The number of $f$ 's at the beginnning of an $L$-block is equidistributed over all integers between 1 and $L$, independent of the choice of the sequence of $(L-1)$-blocks.

(2) Any $K$-block can occur at an allowed position in an $L$-block with the same probability. Thus we can see $T^{n}(x)$ at all allowed positions in all possible $K$-blocks with equal probability. This implies that the distribution of $U$ (on uncoloured names) conditioned on the past is the same as the unconditioned distribution. Hence 
$U$ is a $K$-automorphism. Now we want to show that $T$ is a $K$-automorphism. Since $T^{n}(x)$ can lie in any $K$-block in a $(K+1)$-block with equal probability independent of colours, it follows that $\bigvee_{n}^{n+k} T^{i} \bar{P} / A$ is in a black $K$-block or in a white $K$-block with equal probability. Hence $\bigvee_{-l}^{0} T^{i} \bar{P}$ is $\varepsilon$-independent of $\bigvee_{n}^{n+k} T^{i} \bar{P}$ for any $k$ and any $n>N$.

\section{Rigidity of blocks.}

What we will show here is that the $P$-names ( $\bar{P}$-names) of points have a rigid structure. Therefore any isomorphism between $\left\{T, S_{1}\right\}$ and $\left\{T, S_{2}\right\}$ has to preserve much of the block structure of base names. As usual we want to prove if two names agree in most places, their $n$-blocks are not too far apart. Let $a$ and $b$ be two $n$-block names in the $P$-names of $x$ and $y$. Let $n_{1}$ and $n_{2}$ be integers such that $T^{n_{1}}(x)$ is the first term of $a$ and $T^{n_{2}}(y)$ is the first term of $b$. We say two $n$-blocks, $a$ and $b$, are close if $\left|n_{1}-n_{2}\right|<\sum_{k \leq n} f(k)$. We write $|a-b|=\left|n_{1}-n_{2}\right|$. Let $a^{\prime \prime}$ and $b^{\prime \prime}$ be the (n+ 1)-blocks containing $a$ and $b$ respectively. If $a$ and $b$ are close and of the same order in $a^{\prime \prime}$ and $b^{\prime \prime}$ and if the spacers in $a^{\prime \prime}$ and $b^{\prime \prime}$ are put in the same order, then $a^{\prime \prime}$ and $b^{\prime \prime}$ are close and every $n$-block in $a^{\prime \prime}$ is close to an $n$-block of the same order in $b^{\prime \prime}$. If $a$ and $b$ are close and of the same order in $a^{\prime \prime}$ and $b^{\prime \prime}$ and if the spacers in $a^{\prime \prime}$ and $b^{\prime \prime}$ are put in the reverse order, then $a^{\prime \prime}$ and $b^{\prime \prime}$ are not close, and none of the other $n$-blocks in $a^{\prime \prime}$ is close to an $n$-block in $b^{\prime \prime}$. It is clear from the construction that an $n$-block $a$ in the $\bar{P}$-name of $x$ is close to at most one $n$-block $b$ in the $\bar{P}$-name of $y$ for any $x$ and $y$. If any $(n-1)$-block $a^{\prime}$ in $a$ is close to an $(n-1)$-block $b^{\prime}$ in $b$ and their orders in $a$ and $b$ respectively are different, then it is not hard to see that one of the following cases is true:

(1) Spacers in $a$ and $b$ are in the same order (increasing or decreasing).

(i) if the neighboring $n$-block $\bar{b}$ of $b$, which intersects $a$ has spacers in the same order as $a$, then there is at most one $(n-1)$-block in $a$ that is close to an $(n-1)$-block, which lies in $\bar{b}$.

(ii) if $\bar{b}$ has the spacers in the reverse order from $a$, then there are at most two $(n-1)$-blocks in $a$ that are close to $(n-1)$-blocks which lie in $\bar{b}$.

(2) Spacers in $a$ and $b$ are in the reverse order from each other

(i) If $\bar{b}$ has spacers in the same way as $a$, then there are at most two $(n-1)$-blocks in $a$ that are close to $(n-1)$-blocks in the name of $y$, one of which lies in $b$ and the other in $\bar{b}$.

(ii) If $\bar{b}$ has spacers in the reverse order from $a$, then there are at most three $(n-1)$-blocks in $a$ that are close to $(n-1)$-blocks, one of which lies in $b$ and the others in $\bar{b}$.

All these can be easily shown from the fact that $h(n-1)$ is much bigger than the sum of lengths of spacers and $s(n)$ is bigger than $\sum_{k \leq n} f(k)$. (For details, see [3].) In any case, $a$ has no more than four $(n-1)$-blocks that are close to $(n-1)$-blocks in the name of $y$.

Lemma 3. There exists an $\bar{\varepsilon}>0$ such that if $a$ is an $n$-block in the P-name of $x$, then for any $y$ either 
(1) There is an n-block in the P-name of $y$ close to $a$ and the spacers in $a$ and $b$ are put in the same order; or

(2) There are more than $\bar{\varepsilon} h(n)$ places $i$ in a such that $x_{i} \neq y_{i}$.

Proof. We let $\varepsilon_{1}=1 / h(1)$. Let $\varepsilon_{n-1}$ be the least number such that if (i) is not true, then there are more than $\varepsilon_{n-1} h(n-1)$ places where $x_{i}$ is different from $y_{i}$. We will find $\bar{\varepsilon}$ inductively. There are two cases in which (1) is not true.

(i) There is no $n$-block in the $P$-name of $y$ close to $a$.

(ii) There is an $n$-block, $b$, in the $P$-name of $y$ close to $a$, but the spacers are put in the opposite order.

In both of these cases $a$ has at most four $(n-1)$-blocks close to some (n-1)-blocks in the $P$-name of $y$. Hence there are at least $\left(2^{2 n}-4\right) \varepsilon_{n-1} h(n-1)$ places where $x_{i} \neq y_{i}$. Since $h(n)<\left(2^{2 n}+1\right) h(n-1)$,

$$
\varepsilon_{n} \geq \frac{\left(2^{2 n}-4\right) \varepsilon_{n-1} h(n-1)}{h(n)} \geq \frac{\left(2^{2 n}-4\right) \varepsilon_{n-1} h(n-1)}{\left(2^{2 n}+1\right) h(n-1)} \geq \frac{1-2^{-2 n+2}}{1+2^{-2 n}} \varepsilon_{n-1} .
$$

Since $\prod_{k=1}^{\infty}\left(1-2^{-2 k+2}\right) /\left(1+2^{+k}\right)$ is bounded away from 0 for any $n$, we have (2).

COROLlary 3. Let a be an $n$-block $(n \geq 6)$ in the $\bar{P}$-name of $x$ and let $y$ be any point. Let $K$ be $>h(n) / 2^{n-4}$. If we have $x_{i} \neq y_{i}$ on less than $\bar{\varepsilon} \cdot K / 10$ places for a segment of $K$ consecutive $x_{i}$ 's in $a$, then there exists an $n$-block $b$ in the $\bar{P}$-name of $y$ such that $a$ and $b$ are close and their spacers are put in the same order.

Proof. Since $K>h(n) / 2^{n-4}>2^{n} h(n-1)$, any consecutive $K$-long names will contain many complete $(n-1)$-block names. There is at least one sequence of five consecutive $(n-1)$-blocks each of which has error in less than $\bar{\varepsilon} h(n-1)$ places. (Otherwise at least one fifth of all these $(n-1)$-blocks have error in more than $\bar{E} h(n-1)$ places, which forces error to be bigger than $\bar{\varepsilon} \cdot K / 10)$. This can happen only when the $n$-block $a$ has a close $n$-block $b$ in the $P$-names of $y$ and $a$ and $b$ have spaces in the same order.

What the corollary says is that if two $n$-blocks agree even on a small fraction, then these two blocks must be close. Next we want to show that if two blocks in the $\bar{P}$-name of $x$ and in the $\bar{P}$-name of $y$ agree in most places, then these two blocks have to be of the same colour. For this purpose, we will show that if two blocks in the $\bar{P}$-names agree across a small segment, then 1-blocks have to match very well. This can be done if we let the error in this segment be much smaller than the error in Lemma 3. This is not hard to see because 1-blocks take up at least half of the space.

Lemma 4. Let $a$ and $b$ be two $n$-blocks in the $\bar{P}$-name of $x$ and $y$ respectively. Let $K>h(n) / 2^{n-4}$. Given $\varepsilon>0$, if $x_{i} \neq y_{i}$ in less than $\varepsilon \bar{\varepsilon} K$ places for a segment of $K$ consecutive $x_{i}$ 's in the overlap, then the fraction of 1-blocks in this segment of $K$ consecutive $x_{i}$ 's that are close to 1 -blocks of the same order in $b$ is at least $1-2 \varepsilon$.

Proof. We note here that if an $m$-block in $a$ is not close to an $m$-block of the same order in $b$ and the $(m+1)$-block in $a$ containing this $m$-block is close to the corresponding $(m+1)$-block in $b$, then there is a large error in this $(m+1)$-block. (It could also have spacers in the opposite order from the $(m+1)$-block in $a$ ). If 
the $k$ th $m$-block in $a$ is not close to the kth $m$-block in $b$, then at most one of the $(m-1)$-blocks in this $m$-block is close to an $(m-1)$-block of the same order in the $m$-block in $b$. If the $k$ th $m$-block in $a$ and the $k$ th $m$-block in $b$ have spacers in the opposite order, then there might be one $(m-1)$-block close to an $(m-1)$-block. We cannot have more than one because there is always an odd number of ( $m-$ 1)-blocks in an $m$-block. That is at least $2^{2 m}(m-1)$-blocks in this $m$-block do not have close $(m-1)$-blocks of the same order in $b$. Hence inductively, there are at least $2^{2 m} \cdot 2^{2(m-1)} \cdot 2^{2(m-2)} \cdots 2^{4}$ many 1-blocks which do not have close 1-blocks in this $k$ th $m$-block in $b$. Clearly this is smaller than the number of 1-blocks $\left(\left(2^{2 m}+1\right)\left(2^{2(m-1)}+1\right) \cdots\left(2^{4}+1\right)\right)$ in the $k$ th $m$-block. Also there is at most one $m$-block in the $(m+1)$-block containing this $k$ th $m$-block in $a$ that is close to its corresponding $\boldsymbol{m}$-block in $b$. (We can have such an $m$-block only when $(m+1)$-blocks have spacers in the reverse order.) Let $l$ be the place where the $K$-long consecutive overlap starts. Let $\mathscr{A}$ be the collection of $m$-blocks located between positions $l$, $l+1, \ldots, l+K$ satisfying the following: either they are not close to the corresponding $m$-blocks in $b$ or they have spacers in the reverse order from the corresponding $m$-blocks, but whose $(m+1)$-blocks are close to the corresponding $(m+1)$-blocks in $b$ and these $(m+1)$-blocks have spacers in the same order. Since $a$ and $b$ are close and their spacers are in the same order, any block (in particular, a 1-block) that is not close to its corresponding block in $b$ or whose spacers are in the reverse order from its corresponding block in $b$ is contained in one of these $m$-blocks. In each of these $m$-blocks, by Lemma 3 there exists at least $\bar{\varepsilon} h(m)$ places where names do not match. How many such blocks can we afford in this $K$-consecutive overlap starting at $l$ ?

Since $\sum_{\text {all blocksin } \mathscr{A}} \bar{\varepsilon} h(m)<\varepsilon \bar{\varepsilon} K, \quad \sum_{\text {all blocksin } \mathscr{A}} h(m)<\varepsilon K$. If we let $N$ be the number of 1-blocks in this $K$-consecutive over lap which are not close to their corresponding 1-blocks in $b$, then we know that $N$ is smaller than the total number of 1-blocks in one of these $m$-blocks in $a$. Hence we have $N \cdot h(1)<\sum_{\text {allblocksin } A} h(m)<\varepsilon K$. Because of our choice of $h(1)$ and the number of $f$ s and $s$ 's, there are at least $(K / 2 h(1)) 1$-blocks in any $K$-long sequence. Hence the fraction of good matching 1 -blocks is bigger than

$$
1-\frac{N}{K / 2 h(1)}=1-\frac{\varepsilon K / h(1)}{K / 2 h(1)}=1-2 \varepsilon
$$

Next we want to prove their colours should match, too.

LEMMA 5. There is an $\overline{\bar{\varepsilon}}>0$ such that if $a$ is an n-block in the $\bar{P}$-name of $x$ and $x_{i} \neq y_{i}$ for at most $\overline{\bar{\varepsilon}} h(n)$ places, then there is an $n$-block $b$ in the $\bar{P}$-name of $y$ which is close to $a$ and has spacers in the same order as $a$ and their colours are the same.

Proof. Let $\overline{\bar{\varepsilon}}=\bar{\varepsilon} / 10$. By Lemma 3, we know that $a$ and $b$ are close and they have their spacers in the same order. Also from Lemma 4, at least four-fifths of the 1-blocks in $a$ have to be close to their corresponding 1-blocks in $b$. If two close 1-blocks have different colours, they have errors in at least $\frac{99}{100} h(1)$ places. If $a$ and $b$ have opposite colouring, then all 1-blocks in $a$ and the corresponding 1 -blocks in $b$ have opposite colouring. Then there are at least $\frac{99}{100} h(1) \cdot($ number of 
1-blocks in $a$ which have close 1-blocks in $b$ ) places where their colours are opposite. Hence the error has to be in more than

$$
\frac{99}{100} h(1) \frac{h(n)}{2 h(1)} \frac{4}{5}=\frac{396}{1000} h(n)
$$

places. Therefore $a$ and $b$ have the same colour.

COROLlaRY 4. Let a be an $n$-block $(n \geq 6)$ in the $\bar{P}$-name of $x$ and let $y$ be any point. If we have $x_{i} \neq y_{i}$ in less than $\bar{\varepsilon} K / 4$ places for for a segment of $K$ consecutive $x_{i}$ 's in $a$ where $K>h(n) / 100$ there exists an n-block $b$ in the $\bar{P}$-name of $y$ such that $a$ and $b$ are close and of the same colour and have the same order of spacers.

Proof. This follows immediately from Lemma 5.

\section{Main Theorem}

Let $\varphi$ be an isomorphism between $\left\{T, S_{1}\right\}$ and $\left\{T, S_{2}\right\}$. We will be led to a contradiction as usual [3], [5], [8]. Given $\varepsilon>0$, we can find $N(\varepsilon)$ such that there exists a partition $Q=\left\{Q_{1}, \ldots, Q_{s}\right\}$ each atom of which is a union of atoms in $V_{-N(\varepsilon)}^{N(\varepsilon)} T^{i} \bar{P}$ and $\sum_{i=1}^{5} \mu\left(Q_{i} \Delta \varphi^{-1}\left(\bar{P}_{i}\right)\right)<\varepsilon$. Hence there exists a finite coding $\varphi_{\varepsilon}$ such that the $\bar{P}$-name of $x$ from $-N(\varepsilon)+l$ to $N(\varepsilon)+l$ determines the atom of the partition $Q$ which contains $T^{l} \varphi_{\varepsilon}(x)$. By the ergodic theorem, the $Q$-name and the $\bar{P}_{\text {-name of }} \varphi(x)$ match for all but at most a set of density $\varepsilon$ for almost every point, if we look at long enough names. We fix an $x$ satisfying this property. The following observation will provide the key to the theorem.

Let $a$ be an $n$-block of the $\bar{P}$-name of $x$. We let $\tilde{a}$ be an $n$-block of the $\bar{P}$-name which has the same colour as $a$ and whose base name is the echo name of $a$ (in an abuse of notation, $S_{1}(a)=\tilde{a}$ ). We let $a^{\prime}$ and $\tilde{a}^{\prime}$ be strings of names (not necessarily names of $n$-blocks) mapped by $\varphi_{\varepsilon}$ of $a$ and $\tilde{a}$ respectively. We let $a^{\prime \prime}$ be a string of names whose colours are the opposite of $a^{\prime}$ and whose base names are in the reverse order from the name of $a^{\prime}\left(S_{2}\left(a^{\prime}\right)=a^{\prime \prime}\right)$. If we assume $n$ to be large enough, then $a^{\prime \prime}$ and $\tilde{a}^{\prime}$ have to agree in at least a set of density $1-2 \varepsilon$. (Note that $\varphi S_{1}=S_{2} \varphi$ ). But we will show that this cannot happen using the rigidity of block structures.

Let $\varepsilon$ be $\bar{\varepsilon}^{2} / 100$ where $\bar{\varepsilon}$ is given in Lemma 5. We say an $n$-block $a$ in the $\bar{P}$-name of $x$ is good if

(i) There is an $n$-block $b$ in the $\bar{P}$-name of $\varphi(x)$ that overlaps it in more than $\frac{3}{4} h(n)$ places

(ii) $a$ is not one of the first four or last four $n$-blocks in its $(n+1)$-block

(iii) The $Q$-name of $\varphi(x)$ and the $\bar{P}$-name of $\varphi(x)$ do not match in at most $\varepsilon h(n)$ places.

We also choose $n$ large enough so that $\mu\left(X-X_{n}\right)<1-\varepsilon^{2}$ and $2 N(\varepsilon) / h(n)<\varepsilon^{2}$. We choose $m>n$ such that the fraction of good $n$-blocks in an $m$-block is bigger than $1-\varepsilon$ and every $n$-block appears with proper frequency in the $m$-block.

LEMMA 6. Let $a$ and $b$ be good $n$-blocks in the $\bar{P}$-name of $x$ and $b$ be a translate of $a\left(b=T^{k}(a)\right)$. Let $a^{\prime}$ and $b^{\prime}$ be $n$-blocks of $\varphi(x)$ such that $\left|a-a^{\prime}\right|<\frac{1}{4} h(n)$ and $\left|b-b^{\prime}\right|<\frac{1}{4} h(n)$. Then $\left|T^{k}\left(a^{\prime}\right)-b^{\prime}\right|<\sum_{i \leq n} f(i)$ and $a^{\prime}$ and $b^{\prime}$ have the same colours and their spacers are put in the same order. 
Proof. We note that the $Q$-name of the block $a$ and $b$ are the same. Also since the $Q$-name and the $\bar{P}$-name of $y=\varphi(x)$ differ in a set of at most density $\varepsilon$, two $h(n)$-long sequences of names $\left(y_{l}, y_{l+1}, \ldots, y_{l+h(n)-1}\right)$ and $\left(y_{l+k}, y_{l+k+1}, \ldots, y_{l+h(n)+k-i}\right)$ of $y=\varphi(x)$ differ in at most $2 \varepsilon h(n)$ places. Because of the positions of $a^{\prime}$ and $b^{\prime}$, we have at least $h(n) / 2$-long overlap between the two $n$-blocks $T^{k}\left(a^{\prime}\right)$ and $b^{\prime}$. Hence there is at most $4 \varepsilon h(n)$ error in this overlap. By Corollary $4, T^{k}\left(a^{\prime}\right)$ and $b^{\prime}$ have to be close and of the same colour and their spacers are put in the same order.

We call a sequence $a^{1}, a^{2}, a^{3}, \ldots, a^{k}$ of $n$-blocks in the $\bar{P}$-name of $x$ adjacent if they are contained in one $(n+1)$-block and separated only by spacers. If $a$ is a good $n$-block, then there exists an adjacent sequence $a^{1}, a^{2}, a^{3}, a^{4}$ of $n$-blocks.

THEOREM 6. $\left\{T, S_{1}\right\}$ and $\left\{T, S_{2}\right\}$ are not isomorphic.

Proof. Since there are at most $\varepsilon$ fraction of bad $n$-blocks, there are at most $4 \varepsilon$ fraction of adjacent quadruples which have at least one bad block. (Also if $n$ is sufficiently large, then there exist at least $1-\varepsilon$ fraction of adjacent quadruples all of which are mapped into the same $(n+1)$-block). Let $a, b, c, d$ be an adjacent sequence of good $n$-blocks in the $\bar{P}$-name of $x$, all of which are mapped into the same $(\mathrm{n}+1)$-block in the $\bar{P}$-name of $y=\varphi(x)$. Let $a^{\prime}, b^{\prime}, c^{\prime}, d^{\prime}$ be the $n$-blocks in the $\bar{P}$-name of $y$ which satisfy $\left|a^{\prime}-a\right|<\frac{1}{4} h(n)$ and $\left|b^{\prime}-b\right|<\frac{1}{4} h(n),\left|c^{\prime}-c\right|<\frac{1}{4} h(n)$, $\left|d^{\prime}-d\right|<\frac{1}{4} h(n)\left(a^{\prime}, b^{\prime}, c^{\prime}, d^{\prime}\right.$ are adjacent). Consider good $n$-blocks $\tilde{a}, \tilde{b}, \tilde{c}, \tilde{d}$ in the $\bar{P}$-name of $x$, where $\tilde{a}=a, \tilde{b}=S_{1}(b), \tilde{c}=c, \tilde{d}=d$. We let $\tilde{a}^{\prime}, \tilde{b}^{\prime}, \tilde{c}^{\prime}, \tilde{d}^{\prime}$ be the $n$-blocks in the $\bar{P}$-name of $y$ which satisfy $\left|\tilde{a}^{\prime}-\tilde{a}\right|<\frac{1}{4} h(n),\left|\tilde{b}^{\prime}-\tilde{b}\right|<\frac{1}{4} h(n),\left|\tilde{c}^{\prime}-\tilde{c}\right|<\frac{1}{4} h(n)$, $\left|\tilde{d}^{\prime}-\tilde{d}\right|<\frac{1}{4} h(n)$. We know from Lemma 4 that $\tilde{a}^{\prime}$ has to lie below $\tilde{a}$ within $\sum_{k \leq n} f(k)$ of the way that $a^{\prime}$ lies below $a$. Similarly $\tilde{b}^{\prime}$ below $\tilde{b}, \tilde{c}^{\prime}$ below $\tilde{c}$ and $\tilde{d}^{\prime}$ below $\tilde{d}$. We note that the probability of adjacent quadruples $a, b, c, d$ is the same as that of $\tilde{a}, \tilde{b}, \tilde{c}, \tilde{d}$. The possible configurations of colours of adjacent quadruples all of which are the same $(n+1)$-block are $W W B B, B B W W, W B B W, B W W B$.

Let $b^{\prime \prime}$ be the $n$-block whose base name is the echo of the base name of $b^{\prime}$ and whose colour is the opposite of $b^{\prime}\left(b^{\prime \prime}=S_{2}\left(b^{\prime}\right)\right)$. Since $\tilde{b}=S_{1}(b)$, if we make the proper translation, then the $n$-block name $b^{\prime \prime}=S_{2}\left(b^{\prime}\right)$ differs from the name of $\tilde{b}^{\prime}$ in at most $4 \varepsilon$ places of the overlap which is at least $\frac{1}{4} h(n)$-long. Hence $b^{\prime \prime}$ has to have the same colour as $\tilde{b}^{\prime}$, which in turn implies that $\tilde{b}^{\prime}$ has the opposite colour from $b^{\prime}$. Since $\tilde{a}$ and $\tilde{a}^{\prime}, \tilde{c}$ and $\tilde{c}^{\prime}, \tilde{d}$ and $\tilde{d}^{\prime}$ have same colours, the configuration of colours of the quadruple $\tilde{a}^{\prime}, \tilde{b}^{\prime}, \tilde{c}^{\prime}, \tilde{d}^{\prime}$ is $W B B B, B W W W, W W B W$ or $B B W B$ corresponding to the configurations of colours $a^{\prime}, b^{\prime}, c^{\prime}, d^{\prime}$. But none of these is possible within the same $(n+1)$-block. This completes the proof.

\section{REFERENCES}

[1] W. Ambrose. Representation of ergodic flows. Ann. of Math. 42, 1941, 723-739.

[2] L. Flaminio. An extension of Ratner's rigidity theorem to $n$-dimensional hyperbolic space. Dissertation, Stanford University, 1985.

[3] D. Ornstein. Ergodic Theory, Randomness and Dynamical Systems, Yale University Press, 1974.

[4] D. Ornstein, Personal Communication.

[5] D. Ornstein \& P. Shields. An uncountable family of $\boldsymbol{K}$-automorphisms. Advances in Mathematics, vol. 10, 1973, 63-88. 
[6] K. Park. A $K$-automorphism with two non-isomorphic $Z \times Z_{2}$ actions. Journal D'Analyse Mathématique, vol. 45, 1985, 37-45.

[7] M. Ratner. Rigidity of horocycle flows. Ann. of Math., 115, 1982, 597-614.

[8] D. Rudolph. Non-isomorphic K-automorphisms. Israel J. Math., vol. 23, 1976, 274-287.

[9] D. Rudolph. An isomorphism theory for Bernoulli free $Z$-skew compact group action. Advances in Mathematics, vol. 47, No. 3, 1983, 241-257.

[10] D. Rudolph. Restricted Orbit Equivalence. Mem. Amer. Math. Soc., vol. 54, No. 323, 1985.

[11] R. Zimmer. Algebraic topology of ergodic lie group actions and measurable foliations. Preprint. 